\title{
Comparison of maternal adiposity with neonatal fat mass
}

\author{
A. O’Higgins ${ }^{1}$, A. Doolan ${ }^{2}$, C. O'Connor ${ }^{1}$, M. M. Kennelly ${ }^{1}$, M. Sheridan-Pereira ${ }^{2}$ and M. J. Turner ${ }^{1}$ \\ ${ }^{1}$ UCD Centre for Human Reproduction, Coombe Women and Infants University Hospital, Dublin 8, Ireland and ${ }^{2}$ Trinity \\ College Dublin, Department of Paediatrics, Coombe Women and Infants University Hospital, Dublin 8, Ireland
}

There has been an increase in maternal obesity levels in Ireland and a simultaneous increase in childhood obesity ${ }^{(1,2)}$. Similar trends are emerging throughout Western countries. The Institute of Medicine in the United States of America recently published guidelines recommending targets for gestational weight gain $(\mathrm{GWG})$ with obese women recommended to gain less weight than women with a normal $\mathrm{BMI}^{(3)}$. However there is little evidence available to support that restricting GWG improves obstetrics or neonatal outcomes ${ }^{(3)}$. This prospective study examined the relationship between maternal body composition and neonatal adiposity.

A total of 73 women were recruited to the study during the first trimester of pregnancy. Maternal height was measured in meters to two decimal places using a digital Seca wall mounted meter stick and body composition was measured by bioelectrical impedance analysis (Tanita MC-180). Maternal bioelectrical impedance analysis was repeated at term. Infant body composition was measured within three days of birth using 'infant body composition was measured using air displacement plethysmography (PEA POD infant body composition system, Cosmed, Rome, Italy). Maternal weight change, fat mass change and booking adiposity were compared to neonatal birth weight and neonatal fat mass.

Complete data was available for 57 mother-infant pairs that delivered at term. Of these $50 \%$ had a normal booking BMI, $21 \%$ were overweight and $29 \%$ were obese. Gestational weight gain ranged from 4.1-23.9 kg and was lowest in obese women and highest in women of normal weight. Neonatal fat mass ranged from $19.5 \%$ of body mass to $4.5 \%$ of body mass. Neonatal fat mass was not associated with maternal BMI $(p=0.87)$, maternal fat mass $(p=0.64)$ or gestational weight gain $(p=0.73)$.

Neonatal adiposity is not directly related to maternal body composition nor to maternal weight gain during pregnancy. Restriction of gestational weight gain may not result in neonates achieving normal adiposity levels.

1. Barry S, Fattah C, Farah N, Broderick V, Stuart B, Turner MJ. (2009) The growing challenge of maternal obesity (editorial). IMJ 102, 5-6.

2. Farah N, Maher N, Barry S, Kennelly M, Stuart B, Turner MJ. (2009) Maternal morbid obesity and obstetric outcomes. Obes Facts 2, 352-4.

3. Institute of Medicine. (2009) Weight gain during pregnancy: Re examing the Guidelines. National Academy Press May. 